\title{
Sobrecarga e percepção de qualidade de vida em cuidadores de idosos do Núcleo de Atendimento à Terceira Idade do Exército (Natiex)
}

\author{
Burden and quality of life perception in caregivers of elderly in the Army Geriatric Health Center
}

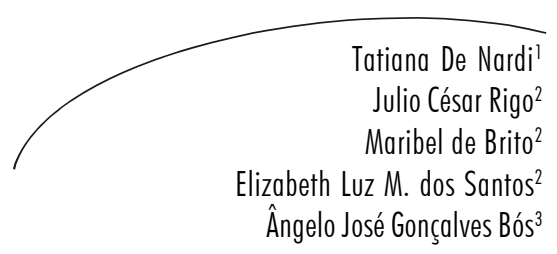

Resumo

Introdução: Dado o envelhecimento populacional, torna-se relevante avaliar a sobrecarga e a qualidade de vida de quem cuida dos idosos. Essa prática costuma ser árdua. $\mathrm{O}$ cuidador precisa acrescentar, às atividades diárias, tarefas referentes ao idoso, expondo-se a um elevado padrão de burden (sobrecarga), ou aspectos negativos associados ao cuidado de dependentes. Isso pode levar a uma piora da qualidade de vida e consequente adoecimento. Objetivos: Avaliar o perfil sóciodemográfico e a relação entre a sobrecarga e a percepção de qualidade de vida de cuidadores de idosos do Núcleo de Atendimento à Terceira Idade do Exército (Natiex). Métodos: 61 participantes foram investigados a partir dos instrumentos WHOQOL-BREF e Zarit Burden Interview. Resultados: Dos 61 cuidadores avaliados, $78,6 \%$ eram do sexo feminino, $75,4 \%$ filhas, $50,8 \%$ casados, $62,3 \%$ com mais de 50 anos e $95,1 \%$ exerciam a atividade sem remuneração. Foi identificada uma relação estatisticamente significativa entre o aumento da sobrecarga e a piora da percepção de qualidade de vida $(\mathrm{p}<0,01)$. Conclusões: À medida que aumenta a sobrecarga, ocorre uma piora da percepção de qualidade de vida em todos os domínios: relações, psicológico, físico e ambiental (domínio ambiente teve o maior impacto). Os resultados encontrados foram superiores a outras amostras brasileiras, possivelmente pela maior facilidade do militar a recursos de saúde. Grupos de apoio a cuidadores devem ser estimulados como prevenção primária das morbidades relacionadas ao cuidar, tendo em vista a demanda crescente de idosos dependentes em nosso meio.
Programa de Pós-graduação em Psicologia, Faculdade de Psicologia. Pontifícia Universidade Católica do Rio Grande do Sul e Núcleo de Atendimento à Terceira Idade do Exército (Natiex). Policlínica Militar de Poto Alegre - Exército Brasileiro. Porto Alegre, RS, Brasil.

2 Núcleo de Atendimento à Terceira Idade do Exército (Natiex). Policlínica Militar de Porto Alegre Exército Brasileiro. Porto Alegre, RS, Brasil.

3 Instituto de Geriatria e Gerontologia. Pontifícia Universidade Católica do Rio Grande do Sul. Porto Alegre, RS, Brasil.

Correspondência / Correspondence

Tatiana De Nardi

Av. João Pessoa, 651 sala 403 - Centro

90040-000- Porto Alegre, RS, Brasil

E-mail: tatydenardi@yahoo.com.br
Palavras-chave: Qualidade de Vida. Cuidadores. Idosos. Perfil de saúde. Burden. 


\section{Abstract}

Introduction: As population ages, it becomes relevant to assess the burden and quality of life of caregivers of elderly. This practice is often difficult. The caregiver needs to add to daily activities, tasks for the elderly, exposing themselves to a high standard of burden or negative aspects associated with the care for dependents. This can lead to a worsening of quality of life and consequent illness. Objectives: To evaluate the socio-demographic profile and the relationship between overload and perceived quality of life of caregivers for the elderly at the Elderly Care Army Center (Natiex). Methods: 61 participants were investigated through the WHOQOL-BREF and Zarit Burden Interview. Results: Of the 61 elderly assessed, $78.6 \%$ were women, $75.4 \%$ were daughters, $50.8 \%$ married, $62.3 \%$ aged over 50 years and $95.1 \%$ had activities without pay. We identified a statistically significant relationship between increased overburden and worsening perception of quality of life $(p<0.01)$. Conclusions: As the burden increases, there is a worsening of the perceived quality of life in all areas: relationships, psychological, physical and environmental (environmental domain showed the greatest impact). The results were higher than other Brazilian samples, possibly due to the easier access of militaries to health care. Support groups for caregivers should be encouraged as a primary prevention of health problems related to care, considering the increasing demand of dependent elderly.
Key words: Quality of Life. Caregivers. Aged. Health Profile. Burden.

\section{INTRODUÇÃO}

A cultura brasileira evidencia a crença de que o cuidado de um idoso deve ser desenvolvido pela própria família em domicílio. Isso é constatado especialmente se o indivíduo dependente for o cônjuge, os pais ou os avós. A literatura refere-se a tal fato como uma naturalização da ideia de família cuidadora. ${ }^{1} \mathrm{Na}$ revisão da literatura, observa-se um predomínio de mulheres exercendo a função de cuidador primário $(73 \%))^{2,3}$

O cuidar de um familiar idoso costuma ser uma tarefa árdua, sobretudo porque geralmente esta tarefa é delegada a apenas uma pessoa, a qual acumula essa atividade a outras de sua rotina pessoal. ${ }^{3}$ Estudos mostram que essa situação interfere na vida do cuidador, pois além de seus compromissos, o mesmo precisa dedicar-se para o desenvolvimento de conhecimento e habilidades referente às necessidades do idoso. ${ }^{4}$ Como resultado, ocorre uma sobrecarga, que muitas vezes leva o cuidador ao adoecimento físico e mental. ${ }^{1,5-8}$

O termo burden vem sendo utilizado para descrever os aspectos negativos associados ao cuidado de pacientes dependentes, ${ }^{9}$ traduzindose como sobrecarga. Esta decorre da necessidade de cuidados ininterruptos, do difícil manejo das manifestações psiquiátricas, somadas a questões emocionais anteriores à instalação da doença. $\mathrm{Na}$ literatura, evidencia-se que cuidadores que pertencem à família nuclear (esposas e filhas) estão ainda mais expostas a um elevado padrão de burden ou sobrecarga, assim como quanto maior o número de meses que o cuidador está nessa atividade, maior a sobrecarga apresentada. ${ }^{9}$

O termo "percepção de qualidade de vida", segundo a Organização Mundial da Saúde (OMS) refere-se à "percepção do indivíduo de sua posição na vida no contexto da cultura e sistema de valores nos quais ele vive e em relação aos seus objetivos, expectativas, padrões e preocupações.” Para avaliação da percepção da qualidade de vida, a OMS desenvolveu um questionário chamado World Health Organization Quality of Life (WHOQOL), em uma primeira versão denominada WHOQOL-100. Em seguida, essa mesma organização, com fins de facilitar e tornar mais breve o processo de investigação da qualidade de vida criou o WHOQOL-BREF. Dados atuais 
sugerem que o WHOQOL-BREF é uma alternativa válida e aplicável em ambiente clínico, sendo uma medida interessante da qualidade de vida especialmente nos domínios físico, psicológico e ambiental. ${ }^{10}$

Recentemente, estudos vêm demonstrando que cuidadores informais ou não profissionais apresentam uma pior percepção de sua Qualidade de vida, quando comparados a cuidadores profissionais. ${ }^{3}$ Além disso, pesquisas mostram que esses tendem a apresentar uma maior sobrecarga, associada à piora da qualidade de vida e muitas vezes acompanhada de depressão. ${ }^{3}$ Apesar disso, no Brasil, ainda são poucas as investigações nessa temática. $\mathrm{O}$ presente estudo buscou investigar o perfil sócio-demografico, a percepção de qualidade de vida de cuidadores de idosos e sua relação com o índice de sobrecarga (burden).

\section{MÉTODO}

Estudo transversal do qual participaram 61 cuidadores de pacientes idosos dependentes. Definiu-se como cuidador a pessoa que assume o papel de cuidar em domicílio ao idoso em situação de dependência e por isso necessita de auxílio em suas atividades gerais da vida diária. ${ }^{4}$ Os critérios de inclusão dos participantes no estudo foram: ser cuidador de idosos com 60 anos ou mais, há um mês ou mais e manifestar, diante do investigador, ser o responsável direto pelos cuidados ao idoso.

Os dados foram coletados através de um questionário autoaplicável sobre o perfil sóciodemográfico estruturado a partir de demandas do serviço e dados da literatura, composto por 20 questões objetivas.

Para informações sobre a percepção de qualidade de vida, foi utilizado o instrumento WHOQOL-BREF, ${ }^{11}$ que é um questionário autoaplicável que aborda os quatro grandes domínios de percepção da qualidade de vida: domínio físico, domínio psicológico, domínio relacional e domínio ambiental. Este é composto de 26 questões, sendo duas gerais (qualidade de vida geral e satisfação com a saúde) e 24 distribuídas entre os domínios físico, psicológico, relações sociais e meio ambiente. ${ }^{10}$

Para avaliação da sobrecarga, foi utilizada a entrevista de sobrecarga Zarit Burden Interview (ZBI). ${ }^{12}$ Esta é uma escala autoaplicável composta de 22 itens, nos quais o participante é avaliado sobre a carga do cuidar na sua vida social, em seu bem-estar físico e emocional e em suas finanças. Apresenta uma graduação de sobrecarga linear de 0 a 74 pontos.

Os cuidadores foram convidados a participar do estudo por chamada telefônica. Posteriormente, assinaram um termo de consentimento livre e esclarecido, seguido da aplicação dos questionários (perfil sóciodemografico, WHOQUOL-bref e ZBI), a qual foi realizada em grupos.

Para a análise dos dados, foi utilizado o software EpiInfo versão 3.5.1. Os dados coletados foram descritos em frequência, com utilização do Teste t. Para comparação entre os índices de sobrecarga da Escala ZBI e os domínios do WHOQOLBREF, foi utilizada análise de variância (ANOVA).

\section{RESULTADOS}

A tabela 1 descreve as características do grupo de cuidadores de idosos estudado. É possível observar um predomínio de mulheres (78,6\%), sendo na maioria filhas $(75,4 \%)$, casadas $(50,8 \%)$ e com mais de 50 anos. O grupo foi estratificado para escolaridade de acordo com ensino fundamental, médio, superior e pós-graduação, sendo o grupo mais prevalente o do ensino médio (49,2\%). Observou-se que apenas $42,7 \%$ dos cuidadores exerciam atividade externa ao domicílio, sendo as mais prevalentes entre os cuidadores as atividades de aposentado, dona de casa ou desempregado. O tempo dedicado à atividade de cuidar foi elevado: $58,4 \%$ referiam cuidar por 18 ou mais horas ou em período 
integral. Foi também identificado que a maioria $(95,1 \%)$ não recebe remuneração especifica para essa atividade e que nunca foram capacitados $(96,7 \%)$ ou tiveram experiência anterior $(86,9 \%)$. Quanto ao apoio recebido para realização da atividade de cuidar, a maior parte dos participantes do estudo menciona não receber apoio psicológico $(75,4 \%)$, nem intercalar a atividade com outras pessoas (78,7\%). A maioria refere não ter apoio religioso (60,7\%). Em relação à prática de atividade física, a maioria $(55,7 \%)$ refere realizar exercícios físicos semanais. Em relação ao perfil da pessoa cuidada, a maioria apresentava oitenta ou mais anos $(63,2 \%)$ e a patologia de conhecimento do cuidador mais prevalente foi a demência de Alzheimer (44,3\%).

Tabela 1 - Perfil sócio-demográfico dos cuidadores familiares e da pessoa cuidada do Núcleo de Atendimento à Terceira Idade do Exército. Porto Alegre, RS, 2010.

\begin{tabular}{|c|c|c|}
\hline Variável & $\mathrm{N}=61$ & Percentual \\
\hline \multicolumn{3}{|l|}{ SEXO } \\
\hline Feminino & 48 & $78,60 \%$ \\
\hline Masculino & 13 & $21,3 \%$ \\
\hline \multicolumn{3}{|l|}{ PARENTESCO } \\
\hline Esposa & 8 & $13 \%$ \\
\hline Filha/o & 46 & $75,4 \%$ \\
\hline Irmão & 2 & $3,3 \%$ \\
\hline Outros & 5 & $8,2 \%$ \\
\hline \multicolumn{3}{|l|}{ ESTADO CIVIL } \\
\hline Casado & 31 & $50,8 \%$ \\
\hline Solteiro & 20 & $32,8 \%$ \\
\hline Separado & 6 & $9,8 \%$ \\
\hline Viúvo & 4 & $6,6 \%$ \\
\hline \multicolumn{3}{|l|}{ IDADE DO CUIDADOR } \\
\hline Até 50 anos & 23 & $37,7 \%$ \\
\hline Entre 50 e 59 anos & 13 & $21,3 \%$ \\
\hline Entre 60 e 85 anos & 25 & $41 \%$ \\
\hline \multicolumn{3}{|l|}{ ESCOLARIDADE } \\
\hline Pós-graduado & 1 & $1,6 \%$ \\
\hline Ensino Superior & 15 & $24,6 \%$ \\
\hline Ensino Médio & 30 & $49,2 \%$ \\
\hline Ensino Fundamental & 15 & $24,6 \%$ \\
\hline \multicolumn{3}{|l|}{ OCUPAÇÃO } \\
\hline Aposentado & 15 & $24,6 \%$ \\
\hline Dona de casa/desempregado & 20 & $32,8 \%$ \\
\hline Trabalham fora & 22 & $36,1 \%$ \\
\hline Trabalham na área da saúde & 4 & $6,6 \%$ \\
\hline \multicolumn{3}{|l|}{ REMUNERAÇÃO } \\
\hline Sim & 3 & $4,9 \%$ \\
\hline Não & 58 & $95,1 \%$ \\
\hline \multicolumn{3}{|l|}{ HORAS/DIA } \\
\hline Até seis horas/dia & 13 & $20,7 \%$ \\
\hline De 7 a 12 horas/dia & 11 & $18,4 \%$ \\
\hline De 13 a 18 horas/dia & 1 & $1,7 \%$ \\
\hline Mais de 18 horas/dia ou integral & 35 & $58,4 \%$ \\
\hline \multicolumn{3}{|l|}{ EXPERIÊNCIA ANTERIOR } \\
\hline $\operatorname{Sim}$ & 8 & $13,1 \%$ \\
\hline Não & 53 & $86,9 \%$ \\
\hline
\end{tabular}


Tabela 1 - Perfil sócio-demográfico dos cuidadores familiares e da pessoa cuidada do Núcleo de Atendimento à Terceira Idade do Exército. Porto Alegre, RS, 2010. (Continuação)

\begin{tabular}{lcc}
\hline Variável & $\mathrm{N}=61$ & Percentual \\
\hline PREPARAÇÃO PARA CUIDAR & & \\
$\quad$ Sim & 2 & $3,3 \%$ \\
$\quad$ Não & 59 & $96,7 \%$ \\
APOIO PSICOLÓGICO & 15 & $24,6 \%$ \\
$\quad$ Sim & 46 & $75,4 \%$ \\
Não & & \\
RELIGIÃO & 24 & $39,3 \%$ \\
Sim & 37 & $60,7 \%$ \\
Não & & \\
INTERCALA COM OUTRA & 13 & $21.3 \%$ \\
PESSOA & 48 & $78,7 \%$ \\
Sim & & \\
Não & 34 & $55,7 \%$ \\
ATIVIDADE FÍSICA & 27 & $44,3 \%$ \\
Sim & & \\
Não & 2 & $3,2 \%$ \\
IDADE DO CUIDADO & 4 & $4,5 \%$ \\
Entre 50 e 59 anos & 17 & $27,8 \%$ \\
Entre 60 e 69 anos & 26 & $42,6 \%$ \\
Entre 70 e 79 anos & 12 & $19,6 \%$ \\
Entre 80 e 89 anos & & \\
Acima de 90 anos & 5 & $8,2 \%$ \\
PROBLEMA DE SAUDE DO & 27 & $44,3 \%$ \\
CUIDADO & 29 & $47,5 \%$ \\
AVC & & \\
Demência & & \\
Outros ou apenas envelhecimento &
\end{tabular}

O desempenho dos cuidadores familiares na escala WHOQOL-BREF nos domínios físico, psicológico, relações e ambiente está descrito na tabela 2. Nela é possível observar um bom grau de percepção de qualidade de vida pelos cuidadores, sendo na maioria satisfatório nos domínios, físico, psicológico e social. Apenas no domínio ambiental foi encontrado um equilíbrio entre os satisfeitos (escore 4) e mais ou menos satisfeitos (escore 3). Foi encontrada diferença estatisticamente significativa entre os escores obtidos por cada domínio, com exceção do escore total, que não apresentou significância estatística. 
Tabela 2 - Descrição dos resultados da escala WHOQOL-BREF nos diferentes domínios. Porto Alegre, RS, 2010.

\begin{tabular}{cccccc}
\hline Escore & Físico & Psicológico & Relações & Ambiental & TOTAL \\
\hline 1 & & & & & 1 \\
& & & & & $(1,6 \%)$ \\
2 & 2 & 4 & 6 & 1 & 2 \\
& $(3,3 \%)$ & $(6,6 \%)$ & $(9,8 \%)$ & $(1,6 \%)$ & $(3,3 \%)$ \\
3 & 26 & 23 & $19(31,1 \%)$ & 29 & 25 \\
& $42,6 \%)$ & $(37,7)$ & & $(47,5 \%)$ & $(41,0 \%)$ \\
4 & 31 & 32 & 33 & 29 & 32 \\
& $(50,8 \%)$ & $(52,5 \%)$ & $(54,1 \%)$ & $(47,5 \%)$ & $(52,5 \%)$ \\
5 & 2 & 2 & 3 & 2 & 1 \\
& $(3,3 \%)$ & $(3,3 \%)$ & $(4,9 \%)$ & $(3,3 \%)$ & $(1,6 \%)$ \\
$\mathrm{p}$ & 0,001 & 0,001 & 0,0386 & 0,002 & 0,0835 \\
\hline
\end{tabular}

$\mathrm{p}=$ ANOVA em relação à diferença entre os grupos de cada quintil.

O índice da percepção de sobrecarga dos cuidadores encontrado no estudo foi médio. Observou-se uma relação inversa entre a qualidade de vida e a sobrecarga, pois à medida que o índice de sobrecarga aumenta, ocorre uma pior percepção da qualidade de vida em todos os domínios.
Conforme descrito na tabela 3, houve diferença significativa nas médias da ZBI entre os quintis do WHOQOL-BREF. A maioria dos domínios apresentou caráter ordinal significativo, pois quanto maior o escore do WHOQOL-BREF em cada domínio, menor a média da ZBI. O domínio relações apresentou uma diminuição gradativa da ZBI, exceto para os últimos dois quintis do WHOQOL-BREF.

Tabela 3 - Média da Escala ZBI em relação à qualidade de vida percebida pelos diferentes domínios do WHOQOL-BREF. Porto Alegre, RS, 2010.

\begin{tabular}{cccccc}
\hline Escore & Físico (DP) & Psicológico (DP) & $\begin{array}{c}\text { Relações } \\
(\mathrm{DP})\end{array}$ & $\begin{array}{c}\text { Ambiental } \\
(\mathrm{DP})\end{array}$ & TOTAL (DP) \\
\hline 1 & & & & 74,0 \\
2 & 54,5 & 38,5 & $41,2(19,09 \%)$ & 74,0 & $44,5(13,44 \%)$ \\
& $(27,58 \%)$ & $(17,67 \%)$ & & $(0,00)$ & \\
3 & 29,2 & 33,4 & $27,2(10,11 \%)$ & 27,9 & $28,4(11,91 \%)$ \\
& $(12,48 \%)$ & $(12,53 \%)$ & & $(11,57 \%)$ & \\
4 & 24,9 & 21,9 & $24,6(12,03 \%)$ & 26,1 & $24,1(10,17 \%)$ \\
& $(10,24 \%)$ & $(10,04 \%)$ & & $(11,01 \%)$ & $18,0(19,80 \%)$ \\
5 & $7,5(4,95 \%)$ & 16,5 & $25,5(16,11 \%)$ & 8,0 & $0,000)$ \\
\hline & & $(17,68 \%)$ & & $(5,66 \%)$ & 0,0002 \\
\hline
\end{tabular}

$\mathrm{DP}=$ Desvio Padrão. 
A figura 1 mostra a relação entre o índice de sobrecarga da ZBI e os escores obtidos nos diferentes domínios do WHOQOL-BREF, apresentando relação inversa entre os índices apresentados. A figura ressalta especialmente a relação entre a diminuição da qualidade de vida no domínio ambiental e o maior aumento no índice de sobrecarga, sendo seguido do domínio físico. Os domínios psicológico e social foram os que provocaram menor aumento no índice de sobrecarga.

\section{Zarit}

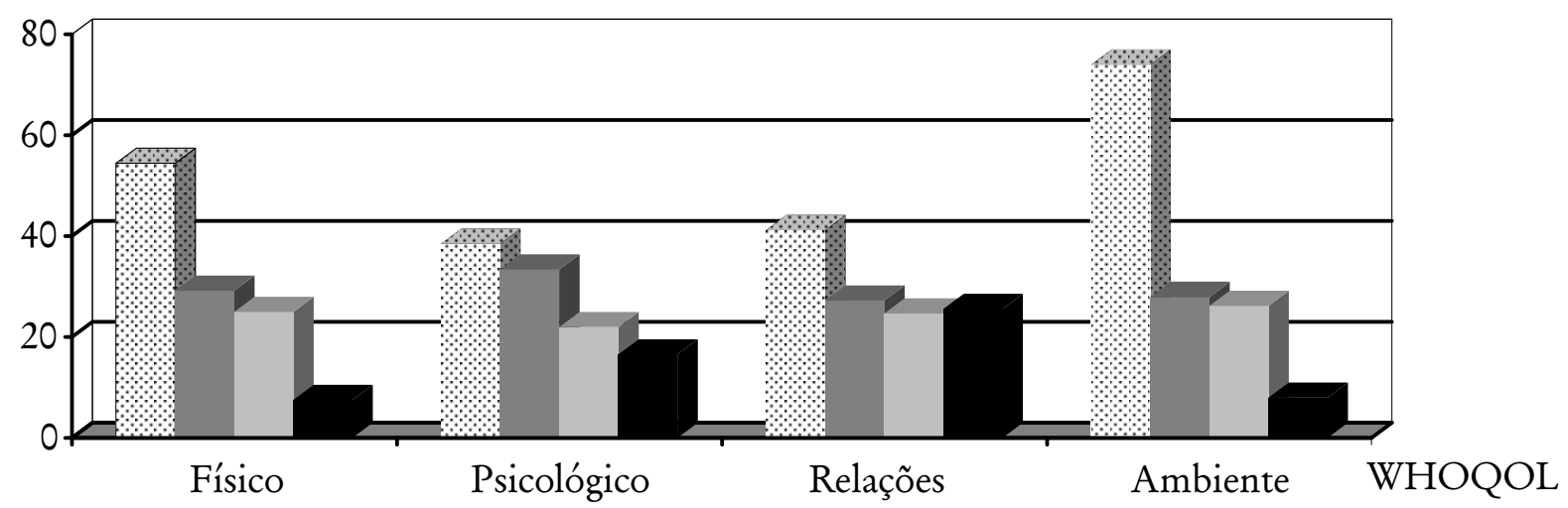

Escore WHOQOL $\square$ Escore WHOQOL $\square$ Escore WHOQOL Escore WHOQOL 5

Figura 1 - Relação entre os índices da Escala ZBI e os escores de percepção de qualidade de vida (WHOQOL-BREF) nos diferentes domínios.

\section{DISCUSSÃO}

O perfil do cuidador do Natiex corrobora os dados de outras pesquisas nacionais, ${ }^{13,14}$ as quais revelam que as mulheres continuam assumindo esse papel com mais frequência. Entretanto, Vilela e colaboradores ${ }^{15}$ consideram que a participação masculina está aumentando, o que é confirmado também por este estudo. Os homens estão aparentemente mais vinculados aos problemas familiares. É possível que isso ocorra devido à igualdade de condições de acesso ao mercado de trabalho por ambos os gêneros. A maior relevância do salário feminino em algumas famílias faz com que mais mulheres prossigam exercendo suas profissões. Este índice também está associado ao maior número de internações geriátricas. Uma vez que o homem e a mulher precisam trabalhar fora, esta, em alguns casos, acaba sendo a opção.

Uma tendência observada na literatura é o fato de a maior parte dos cuidadores serem também idosos. ${ }^{8}$ Neste estudo se observou que a maioria é aposentada ou desempregada, possivelmente pela maior disponibilidade de tempo. Além disso, na realidade brasileira, as pessoas mais jovens têm maior acesso ao mercado de trabalho, assim as famílias optam que estas permaneçam ativas e aumentem a renda familiar. Outra questão que se observa na presente investigação e se relaciona com a maior expectativa de vida no Brasil, ${ }^{16}$ é a faixa etária elevada dos idosos dependentes, cujos filhos e cônjuges já se encontram também em idade avançada. Este um fator de risco para o cuidador, uma vez que o torna mais vulnerável quando exposto ao estresse e a esforços físicos elevados.

À semelhança de outros estudos com cuidadores, ${ }^{14,15,17}$ observa-se que o processo de cuidar do idoso pode desencadear limitações no cotidiano do cuidador familiar, dedicado permanentemente ao idoso e com pouco tempo para realizar outros papéis, como atividades da vida social, de trabalho remunerado ou mesmo para o autocuidado. 
Os cuidadores deste estudo apresentaram pouco apoio psicológico, religioso ou de auxílio no processo de cuidar. Como consequência, vivem em uma intensa rotina, que sobrecarrega o indivíduo e muitas vezes leva ao adoecimento. ${ }^{15}$ Estudos revelam a importância de se ter a colaboração de outros membros da família. O auxílio em poucas tarefas, ou mesmo por meio de visitas, já será percebido como uma forma de apoio. Sentir-se sozinho diante da exposição prolongada a situações altamente estressantes é um dos fatores que mais contribui para o esgotamento geral do indivíduo. ${ }^{18}$ A sensação de dividir com outras pessoas o desgaste provocado pela necessidade de enfrentamento dos eventos negativos cotidianos suaviza o impacto provocado pelo excesso de tarefas. ${ }^{8}$

Os resultados desta investigação confirmam que quanto maior o índice de sobrecarga (ZBI), pior será a percepção de qualidade de vida em todos os domínios da qualidade de vida, o que já está documentado pela literatura. ${ }^{4,15}$ Entretanto, observase maior evidência dessa relação no domínio ambiental, o que sugere que o aumento da sensação de sobrecarga está relacionado a uma piora das condições do ambiente, englobando finanças, moradia, transporte e recursos básicos. Cabe ressaltar que esses participantes obtiveram escores de sobrecarga melhores do que a maioria dos estudos brasileiros, ${ }^{4}$ possivelmente devido a população estudada estar vinculada ao serviço de saúde do Exército, o que traduz maior facilidade de acesso à assistência devido à renda fixa e plano de saúde.

Estudos referem o fato de o cuidador não exercer atividade remunerada como um fator de risco para sobrecarga. ${ }^{3}$ Conflitos familiares podem surgir decorrentes das questões financeiras, especialmente quando o cuidador precisa utilizar a renda do idoso dependente para suas necessidades. Decisões em relação a gastos também são consideradas potenciais geradores de sobrecarga, uma vez que as medicações, demandas médicas e de adaptação ambiental elevam as despesas mensais.

Evidencia-se que a realização de grupos de apoio e de educação são importantes ferramentas para ampliar as possibilidades de o cuidador lidar com os desafios dessa atividade. A partir da identificação de estratégias de outras pessoas que passaram pela mesma situação e da orientação técnica, temos possibilidade de tornar esse trabalho mais eficaz e com menor sobrecarga. É essencial pensar em modos de possibilitar um nível satisfatório de qualidade de vida no domínio ambiental. Uma vez que o cuidador fica eventualmente impedido de trabalhar, esse domínio pode ficar diretamente afetado, sobretudo pela perda financeira. Estratégias de remuneração do cuidador e de ampliação de recursos públicos para o apoio dessa população são importantes e ampliariam a possibilidade de manutenção da saúde desses indivíduos. ${ }^{6,13}$

Enfim, algumas limitações são observadas neste estudo, especialmente no que tange à avaliação de psicopatologias e demais doenças atuais. Uma vez observada a sobrecarga emocional e uma redução na percepção de qualidade de vida, seria relevante a realização de investigação clínica que esclarecesse as comorbidades que podem estar relacionadas a esses achados.

\section{CONCLUSÕES}

À medida que aumenta a sobrecarga, ocorre uma piora da percepção de qualidade de vida em todos os domínios: relações, psicológico, físico e ambiental, tendo o último o maior impacto. As questões ambientais, como condições de moradia, de alimentação e meio de transporte, são importantes fatores relacionados à sensação de sobrecarga. Os dados do Natiex foram melhores que os de outras amostras brasileiras, possivelmente pela facilidade de acesso a recursos, devido à renda fixa e assistência à saúde.

Grupos de apoio e orientação a cuidadores tornam-se fundamentais para a qualidade de vida desses indivíduos e devem ser estimulados como prevenção primária das morbidades relacionadas ao cuidar.

Enfim, o envelhecimento populacional e a consequente demanda de cuidadores tornam mais indivíduos vulneráveis à sobrecarga e ao comprometimento da qualidade de vida. 


\section{AGRADECIMENTOS}

À Policlínica Militar de Porto Alegre: diretor, militares e funcionários civis que colaboraram e disponibilizaram recursos materiais e físicos para realização deste estudo.

Aos participantes voluntários que incentivaram a pesquisa nesta Instituição.

\section{REFERÊNCIAS}

1. Sena ELS, Gonçalves LHT. Vivências de familiares cuidadores de pessoas idosas com doença de alzheimer : perspectiva da filosofia de merleau-ponty. Text Context Enferm 2008; 17(2): $230-40$.

2. Cerqueira ATAR, Oliveira NIL. Programa de apoio a cuidadores: uma ação terapêutica e preventiva na atenção à saúde do idoso. Psicol USP 2002; 13(1): 1-10.

3. Takahashi M, Tanaka K, Miyaoka H. Depression and associated factors of informal caregivers versus professional caregivers of demented patients. Psychiatry and Clinical Neurosciences 2005; 59 (4): 473-80.

4. Amedola F, Oliveira MAC, Alvarenga MRM. Qualidade de vida dos cuidadores de pacientes dependentes no programa de saúde da família. Text Context Enferm 2008; 17(2): 266-72.

5. Nascimento L, et al. Cuidador de idosos: conhecimento disponível na base de dados LILACS. Rev Bras Enferm 2008. 61(4): 514-17.

6. Schossler T, Crossetti MG. Cuidador domiciliar do idoso e o cuidado de si: uma análise através da teoria do cuidado humano de jean watson. Text Context Enferm 2008; 17(2): 280-87.

7. Inouye K, Pedrazzini ES, Pavanini SCI. Octagenários e Cuidadores: perfil sóciodemográfico e correlação da variável qualidade de vida. Text Context Enferm 2008; 17(2): 350-57.

8. Luzardo AR, Gorini MIPC, Silva APSS. Características de idosos com doença de alzheimer e seus cuidadores: uma série de casados em um serviço de neurgeriatria. Text Context Enferm 2006, 15(4): 587-94.

9. Garrido R, Menezes PR. Impacto em cuidadores de idosos com demência atendidos em um serviço psicogeriátrico. Rev. Saúde Pública 2004 dez; 38 (6): 835-41

10. Carrol RE, et al. A comparison of the whoqol100 and the whoqol-bref in detecting change in quality of life. Quality of life research 2000; 9: 121-24.

11. Fleck MPA. Versão em português do instrumento de avaliação de qualidade de vida (WHOQOL): whoqol Abreviado-Versão Em Português. 1998. [ Acesso em 28 out 2008].Disponível Em: < Http:// Www.Ufrgs.Br/ Psiq/Whoqol84.Html $>$.

12. Zarit SH, Reever KE, Bach-Peterson J. Relatives of the impaired elderly correlates of feelings of burden. Gerontologist 1980; 20(6): 649-55.

13. Rodrigues MR, Almeida RT. Papel do responsável pelos cuidados à saúde do paciente no domicílio : um estudo de caso. Acta Paulista Enferm 2005; 18(1): 20-24.

14. Karsch UM. Idosos dependentes: famílias e cuidadores. Cad. Saúde Pública 200319 (3): 861-66.

15. Vilela $A B$, et al. Perfil do familiar cuidador de idoso doente e/ou fragiizado do contexto sociocultural de Jequié-BA. Rev Bras Geriatr Gerontol 2006; 9(1):55-69.

16. Chaimowicz FA. Saúde dos idosos brasileiros às vésperas do século XXI: problemas, projeções, alternativas. Rev Saúde Pública 1997; 31(2):184200.

17. Luzardo AR, Waldman BF. Atenção ao familiar cuidador do idoso com doença de Alzheimer. Rev. Acta Scientiarum 2004 jan/jun; 26 (1): 135-45.

18. Caldeira APS, Ribeiro RCHM. O

Enfrentamento do Cuidador do Idoso com Alzheimer. Arq Ciênc Saud 2004; 11 (2): 2-6. 